\title{
Phages \& antibiotic resistance: are the most abundant entities on earth ready for a comeback?
}

\author{
Colin Hill*,1, Susan Mills ${ }^{1}$ \& Reynolds P Ross ${ }^{1}$ \\ ${ }^{1}$ APC Microbiome Ireland \& School of Microbiology, University College Cork, Cork, Ireland \\ *Author for correspondence: c.hill@ucc.ie
}

Bacteriophages, which lost out to antibiotic therapy in the past, may be poised to make a comeback. Once discarded because of their narrow activity spectrum, it can now be viewed as a major advantage that these intracellular, self-replicating entities can exert their killing effect with minimal damage to the commensal microbiome. In eastern Europe, phages continue to be used both prophylactically and therapeutically to treat infections. More recently, much needed regulated clinical trials are underway with a view to restoring phage therapy as a tool for mainstream medicine, although current regulations may impede their full potential. One hundred years after their discovery, and amid an antibiotic resistance crisis, we must ask, what can be done to harness their full antibacterial potential?

First draft submitted: 6 November 2017; Accepted for publication: 18 December 2017; Published online: 24 May 2018

Keywords: antibiotic resistance $\bullet$ bacteriophage $\bullet$ clinical trials $\bullet$ Escherichia coli $\bullet$ phage resistance $\bullet$ Pseudomonas aeruginosa • Staphylococcus aureus

\section{Bacteriophages: a brief history}

When bacteriophages (phages) were first described 100 years ago, their potential applications in the treatment of infectious disease were immediately obvious [1]. The discovery of a self-replicating antimicrobial capable of killing pathogenic bacteria was potentially transformative to human health. Of course, the advent of efficacious broad-spectrum antibiotics essentially put a stop to phage therapy as a viable option. One of the central issues was the narrow host range of most phages (restricted to the strain level in many instances) and the associated need for an arsenal of phages and knowledge of the infecting strain for effective application. In addition, phages are complex biological structures which must be propagated on a suitable host. They are often much simpler to produce than small-molecule antibiotics, although the latter can be administered essentially in ignorance of the identity of the infectious agent. In most parts of the world, the use of antibiotics was seen as a cure all, leading to a complacency surrounding infectious disease which lasted for decades. Many sources have attributed a 1969 statement to the US surgeon general, Dr William Stewart that 'it is time to close the book on infectious diseases, and declare the war against pestilence won.' While the accuracy of this attribution has been questioned [2], it does give a sense of the prevailing attitude in medical circles at that time. In a press release in July 2017, the WHO reported that antibiotic-resistant Neisseria gonorrhoeae, the causative agent of the sexually transmitted disease gonorrhea, was on the rise with highincome countries reporting cases of untreatable infection by all known antibiotics [3]. Antibiotic resistance has been observed in many common pathogens [4] and in the USA and Europe antibiotic-resistant bacteria annually result in almost 50,000 deaths [5].

A more recent development has been our growing awareness of the importance of the microbiome to human and animal health. Several studies describe the devastating impact of broad-spectrum antibiotics on the microbiome, with unknown but potentially lifelong consequences for the host [6,7]. Clostridium difficile-associated diarrhea could essentially be described as one of the first 'microbiome-based' diseases in that it is usually initiated by antibiotic-induced damage to the microbiome, with devastating consequences for the human host [8]. That which was perceived as the primary drawback of phages - their narrow spectrum - may well now be their greatest asset 


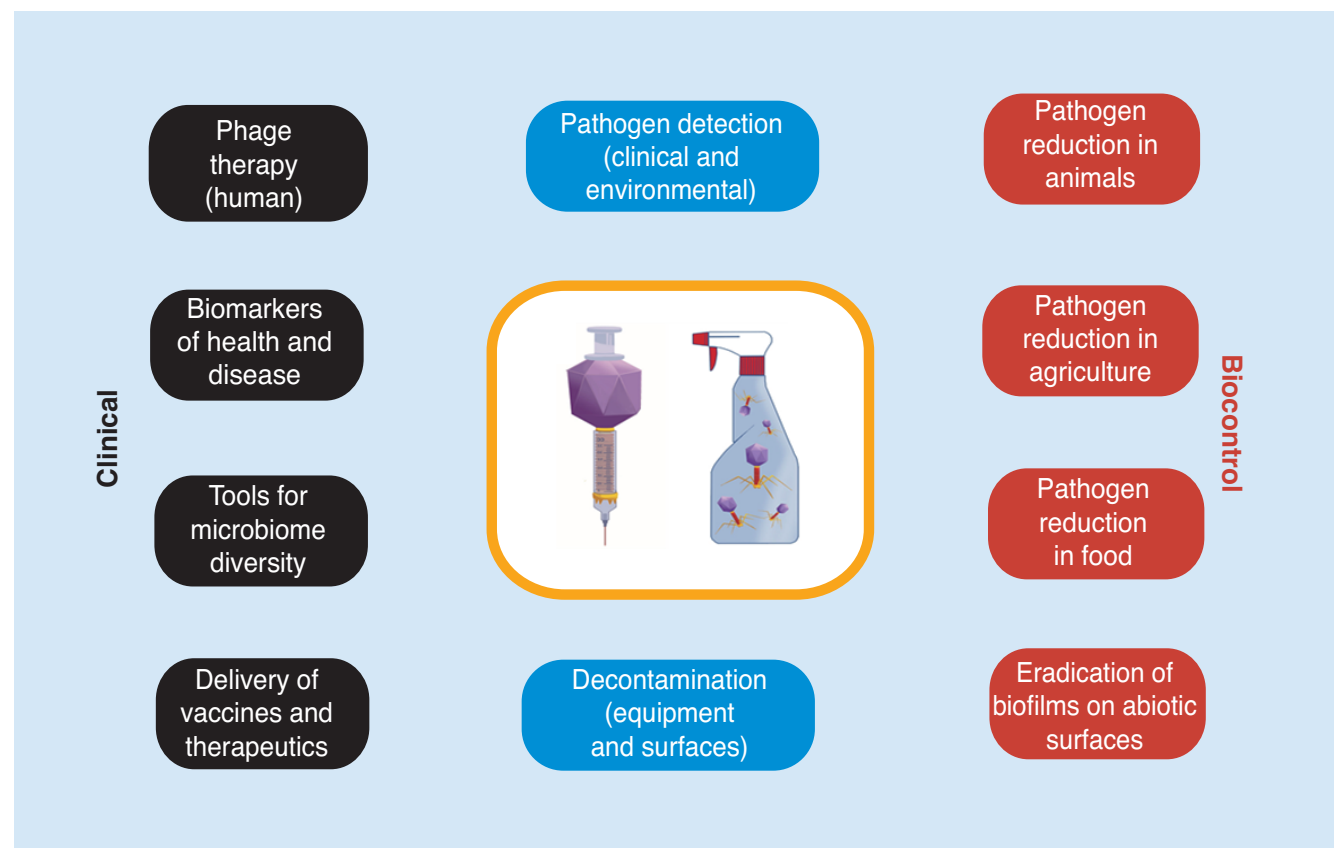

Figure 1. Range of clinical and biocontrol applications of phages.

in a situation where we want to treat infectious disease without inflicting collateral damage to our commensal microbiomes.

In the West, phages achieved significance not as therapeutic agents, but as tools essential to the discovery of many fundamental concepts in biology [9]. Hershey and Chase used phages to identify DNA as the material of genes in 1952 [10], while further pioneering studies paved the way to our understanding of gene regulation [9]. Phage research has been crucial in establishing many of the central doctrines of molecular biology and has given rise to key molecular biology reagents including restriction enzymes, DNA ligase and more recently the Clustered Regularly Interspaced Short Palindromic Repeat (CRISPR)-Cas system that has provided a general approach to genetically manipulating genomes [9].

Never has the time been so appropriate to revisit phages as potential therapeutics in the face of the antibiotic resistance crisis, and during the growing need for targeted antimicrobials which do not result in collateral microbiome damage. Perhaps with the benefit of hindsight, we can learn from our mistakes that have led to the current antibiotic crisis, and deploy phages in a more precise and defined manner to fight against the increasing burden of infection (Figure 1). Fortunately, we do not have to speculate about how phages might have been used to combat disease because we have a practical example of where they have been used - eastern Europe.

\section{The status of phage therapy in eastern Europe \& the western world}

Phage therapy is practiced today in several places around the globe including Russia, Georgia and Poland, the latter of which has a government-approved phage treatment center. However, most of the available information stems from the latter two countries. Phage therapy is routinely used in Georgian hospitals and clinics for therapeutic and prophylactic purposes; primarily driven by research and development emanating from the Eliava Institute in Tbilisi. The Institute's main focus has been on the development of phage cocktails, along with their characterization, production and implementation for human therapy and prophylaxis [11,12], veterinary applications, food safety, and environmental protection [12]. Over-the-counter phage preparations for self-care or for use on the advice of a doctor are commercially available throughout Georgia, while medical personnel have access to a broader range of phage therapy products [11]. Cocktails are updated regularly by testing against current problematic strains and upgrading as necessary with new phages [13]. In 2003, the Phage Therapy Center was founded with a mission to provide therapy for difficult-to-treat infections, including acute and chronic infections, antibiotic resistant infections and infections in patients with poor circulation. This multifaceted center works directly with patients, manages patient 
records, tests the effectiveness of cocktails, and when required, arranges the isolation of new phages by the Eliava Institute.

The Hirszfeld Institute of Immunology and Experimental Therapy, which was established in 1952 by the Polish Academy of Sciences in Wroctaw, has collected over 800 phages against many pathogens [14]. The Institute isolates and identifies bacterial strains from patient specimens, determines phage sensitivity of isolated strains against its phage collection and prepares specific phage lysates for treatment. Hence, their 'personalized' approach differs to that of the Eliava Institute which uses a 'pre-prepared cocktail' [11]. Following Poland's membership entry to the European Union, the government-approved Phage Therapy Unit (an outpatient clinic) was established in 2005 to enable the continued application of phage therapy under EU regulations and is supported by the Bacteriophage Laboratory of the Hirzfeld Institute. The Unit deals with cases where prior antibiotic treatment has failed and is only possible under the rules of a 'therapeutic experiment' (experimental treatment) in line with respective Polish regulations and the Declaration of Helsinki [15].

However, despite the apparent success of phage therapy in these locations, the paucity of peer-reviewed controlled clinical trials makes it difficult to properly evaluate the effectiveness of such therapeutics by western standards; although peer-reviewed clinical data generated from observational studies of phage therapy in patient treatment are available [16-26] (see Table 1 for details).

The 'pre-prepared cocktail' approach of the Eliava Institute involves complex mixtures of unknown phages, which would fail to gain acceptance as human therapeutics under the EMA and US FDA regulations. Indeed, the phage populations present in the Eliava Institute's IntestiPhage cocktail were revealed in 2015 by metagenomic analysis that identified 23 major phage clusters in different abundances, mainly composed of Myoviridae (35\%), Siphoviridae (32\%) and Podoviridae (15\%) [13]. While no undesirable genes were identified, the authors suggest that low abundance phages were potentially missed in the analysis.

Unlike conventional medicines, the success of phage therapy relies on the ability to update cocktails with different phages or to develop personalized phage preparations to treat specific infections that are beyond the scope of current EU regulatory frameworks. Indeed, at a 2015 EMA workshop [27], it was clarified that phage therapy falls under the existing EU regulatory framework on human medicinal products in Directive 2001/83/EC [28]. Thus, prior to patient use these products require marketing authorization that is based on 'pharmaceutical, preclinical and clinical documentation' [29]. However, developing a personalized phage therapy product or updating a preexisting phage cocktail which already has a marketing authorization will result in the need for a new or separate marketing authorization, to ensure the safety, quality and efficacy of the product, which is a complex and time-consuming process, thus immediately limiting the scope of phage therapy and in most cases rendering it ineffective where immediate treatment is required. Fauconnier [30] suggests the need for a novel regulatory framework to address the issue of customized phage therapy medicinal products in the form of a 'biological master file,' a procedure that is currently in existence for chemical drugs but not for biologically active substances. In this regard, regulatory approval could be sought for each individual phage, addressing issues of Good Manufacturing Practices as well as quality and safety issues. The final phage therapy product would be prepared as a magistral formula that refers to 'medicinal products prepared in a pharmacy in accordance with a medical prescription for an individual patient,' which does not require registration as it is beyond the scope of current European medicine legislation. Such an approach would require trained personnel with the ability to rapidly identify the infectious agent(s) and determine phage specificity prior to preparation of the formula, suggesting the need, at the very least, for dedicated phage therapy booths in pharmacies or perhaps phage therapy centers such as those which have been established in Georgia and Poland.

While personalized medicine is not a new field of therapeutics, it is a growing field with potential to save many lives. In this regard, new regulatory processes will undoubtedly come into existence that will continue to ensure the quality and safety of personalized treatments but without compromising therapeutic efficacy. When this will happen for phage therapy remains to be seen and is undoubtedly subject to the outcome of new and ongoing clinical trials. However, as we will see in the following section, performing clinical trials with phage(s) under the current system can be fraught with unanticipated regulatory and biological issues and while solutions continue to be sought, successful trials are an essential prerequisite to the introduction of phage therapy to conventional medicine. 


\begin{tabular}{|c|c|c|c|c|c|}
\hline Disease & Target organism & $\begin{array}{l}\text { Number of } \\
\text { patients }\end{array}$ & Route & Details and outcome & Ref. \\
\hline Ulcers and wounds & $\begin{array}{l}\text { Proteus } \\
\text { Escherichia coli } \\
\text { Staphylococcus aureus } \\
\text { Staphylococcus } \\
\text { epidermidis } \\
\text { Pseudomonas } \\
\text { aeruginosa } \\
\text { Streptococcus }\end{array}$ & $\mathrm{n}=96$ & $\begin{array}{l}\text { PhageBioDerm, } \\
\text { wound-healing } \\
\text { preparation }\end{array}$ & $\begin{array}{l}\text { In } 67 \text { of } 96 \text { patients, wounds/ulcers completely healed. } \\
\text { Microbiological data available for } 22 \text { patients revealed that } \\
\text { healing was associated with elimination or reduction of } \\
\text { pathogens }\end{array}$ & [16] \\
\hline Septicemia & $\begin{array}{l}\text { S. aureus } \\
\text { P. aeruginosa } \\
\text { E. coli } \\
\text { Klebsiella pneumoniae } \\
\text { Above pathogens and } \\
\text { Proteus mirabilis, } \\
\text { Morganella morganii, } \\
\text { Enterobacter }\end{array}$ & $\begin{array}{l}\mathrm{n}=15 \\
\mathrm{n}=8 \\
\mathrm{n}=7 \\
\mathrm{n}=3 \\
\mathrm{n}=61\end{array}$ & $\begin{array}{l}\text { Oral, three-times a day } \\
\text { after neutralization of } \\
\text { gastric juices }\end{array}$ & $\begin{array}{l}\text { Complete recovery in } 80 \text { patients. Phage therapy was } \\
\text { ineffective in } 14 \text { patients. No significant difference reported } \\
\text { for phage therapy alone or phage therapy }+ \text { antibiotics in } \\
\text { terms of outcome of phage treatment }\end{array}$ & [17] \\
\hline $\begin{array}{l}\text { Infected local radiation } \\
\text { injuries }\end{array}$ & S. aureus & $\mathrm{n}=2$ & $\begin{array}{l}\text { PhageBioDerm, } \\
\text { (wound-healing } \\
\text { preparation containing } \\
\text { phages and ciprofloxacin) }\end{array}$ & $\begin{array}{l}\text { Within 2-7 days, purulent drainage stopped, within } 7 \text { days } \\
\text { pathogen was eliminated and clinical symptoms improved }\end{array}$ & [18] \\
\hline Urinary tract infection & MRSA & $\mathrm{n}=1$ & Oral, three-times a day & $\begin{array}{l}\text { GI tract of patient colonized with MRSA and patient } \\
\text { suffering from urinary tract infections caused by same } \\
\text { pathogen. Treatment with phages resulted in complete } \\
\text { eradication of pathogen }\end{array}$ & [19] \\
\hline & $\begin{array}{l}\text { MRSA } \\
\text { MRCNS } \\
\text { MSSA }\end{array}$ & $\begin{array}{l}\mathrm{n}=2 \\
\mathrm{n}=1 \\
\mathrm{n}=3\end{array}$ & $\begin{array}{l}\text { Oral, three-times a day } \\
\text { after neutralization of } \\
\text { gastric juices and/or } \\
\text { locally }\end{array}$ & $\begin{array}{l}\text { Study highlights significant economic savings of phage } \\
\text { therapy in terms of healthcare costs compared with } \\
\text { conventional treatment }\end{array}$ & [20] \\
\hline $\begin{array}{l}\text { Chronic bacterial } \\
\text { prostatitis }\end{array}$ & Enterococcus faecalis & $\mathrm{n}=3$ & Rectal application & $\begin{array}{l}\text { Pathogen eradication, abatement of clinical symptoms and } \\
\text { lack of early disease recurrence }\end{array}$ & [21] \\
\hline Corneal abscess & S. aureus & $\mathrm{n}=1$ & Topical and intravenous & Eradication of pathogen and stabilization of ocular signs & [22] \\
\hline $\begin{array}{l}\text { Necrotizing } \\
\text { pancreatitis }\end{array}$ & $\begin{array}{l}\text { Multidrug-resistant } \\
\text { Acinetobacter } \\
\text { baumannii }\end{array}$ & $\mathrm{n}=1$ & $\begin{array}{l}\text { Intracavitory and } \\
\text { intravenous }\end{array}$ & Eradication of pathogen and patient's return to health & [23] \\
\hline $\begin{array}{l}\text { Septicemia and acute } \\
\text { kidney injury }\end{array}$ & $\begin{array}{l}\text { P. aeruginosa } \\
\text { (colistin-only-sensitive) }\end{array}$ & $\mathrm{n}=1$ & $\begin{array}{l}\text { Intracavitory and } \\
\text { intravenous }\end{array}$ & $\begin{array}{l}\text { Pathogen eradicated from blood, C-reactive protein levels } \\
\text { dropped, fever disappeared and kidney function returned } \\
\text { after a few days }\end{array}$ & [24] \\
\hline Chronic otitis & P. aeruginosa & $\mathrm{n}=24$ & $\begin{array}{l}\text { Phage preparation added } \\
\text { to ear }\end{array}$ & $\begin{array}{l}\text { Phase I/II controlled clinical trial: phage-treated group } \\
\text { reported statistically significant clinical improvements from } \\
\text { baseline compared with the control group }\end{array}$ & [38] \\
\hline Leg ulcers & $\begin{array}{l}\text { P. aeruginosa } \\
\text { S. aureus } \\
\text { E. coli }\end{array}$ & $\mathrm{n}=39$ & Local & $\begin{array}{l}\text { Phase I safety trial: no adverse effects were reported. No } \\
\text { significant difference was reported between the } \\
\text { phage-treated group and the control group }\end{array}$ & [39] \\
\hline $\begin{array}{l}\text { Prophylactic use of } \\
\text { phage therapy for } \\
\text { respiratory infections }\end{array}$ & - & $n=510$ & - & $\begin{array}{l}\text { Controlled trial: phage therapy was used in conjunction } \\
\text { with bitilisin- } 5 \text { and resulted in reduction of incidence of } \\
\text { respiratory infection, tonsillitis and other respiratory } \\
\text { diseases in subjects }\end{array}$ & [115] \\
\hline Childhood diarrhea & E. coli & $n=120$ & Oral & $\begin{array}{l}\text { Phase II controlled clinical trial: phage therapy did not } \\
\text { improve diarrhea outcome above the standard treatment }\end{array}$ & [54] \\
\hline
\end{tabular}

\section{Phages \& infectious disease}

Despite the lack of controlled clinical trials, it is believed that phage therapy has provided enough evidence in clinical practice [11] to warrant further investigation through regulated clinical trials to establish it in the 'realms of evidence-based medicine' [31]. Such trials require completion in accordance with FDA or EMA guidelines of which the gold standard is double-blind, randomized-controlled multicenter (international) trials [32]. Three human pathogens, Pseudomonas aeruginosa, Escherichia coli and Staphylococcus aureus, are the focus of recent and ongoing trials. 


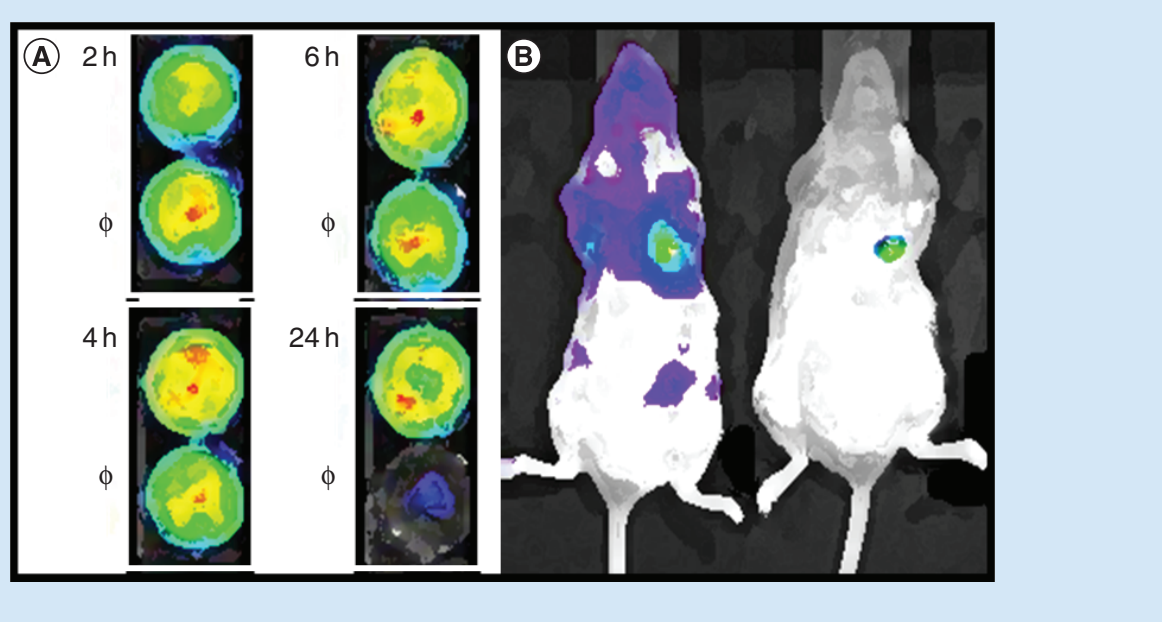

Figure 2. Phage elimination of Pseudomonasaeruginosa. (A) Light emitted from Lux-tagged $P$. aeruginosa grown on a cystic fibrosis bronchial epithelial cell line for $24 \mathrm{~h}$ in the presence (bottom well) and absence (top well) of two lytic phages isolated from a wastewater treatment plant. (B) Phages administered $2 \mathrm{~h}$ after mice were infected with $P$. aeruginosa are seen to clear the infection in the test mouse (far right). Control mice received no phages.

Reproduced with permission from [37].

\section{P. aeruginosa}

P. aeruginosa is a ubiquitous Gram-negative microorganism found in soil and water and can be isolated from plants, animals and humans but it is also an opportunistic pathogen which can cause a wide variety of hospital acquired infections, particularly in individuals with compromised immune systems, chronic pulmonary infections such as cystic fibrosis and open wounds [33]. Its metabolic diversity and ability to tolerate a variety of physical conditions through biofilm formation enables it to survive in community and hospital settings [34]. The pathogen has exhibited resistance against a range of antibiotics including members of the fluoroquinolones, $\beta$-lactams and aminoglycosides with multidrug-resistant varieties expressing resistance to three or more drug classes; achieved through the acquisition of resistance genes or chromosomal mutations [34]. Failure of antibiotic treatments has also been attributed to its ability to form biofilms [35]. Phages against $P$. aeruginosa have been isolated from settings such as hospital sewage, seawater, ponds and rivers [36] and a waste water treatment plant [37] (Figure 2).

The success of $P$. aeruginosa phages for the treatment of human ailments has been validated in several case studies and in a small number of clinical and preclinical trials [11]. In 2009, Wright et al. reported the results of a randomized, double-blind, placebo-controlled, Phase I/II clinical trial using phages to treat antibiotic-resistant $P$. aeruginosa in chronic otitis [38]. The phages were prepared by Biocontrol Limited, a British phage therapy firm and the trial was approved by the UK Medicines and Healthcare Products Regulatory Agency and the Central Office for Research Ethics Committees ethical review process. The trial included 24 patients, aged 2-58, infected with antibiotic-resistant $P$. aeruginosa with reported sensitivity to one or more of the six phages in the phage preparation (Biophage-PA). Twelve patients were treated with a single dose of Biophage-PA (containing $10^{5}$ pfu of each of the six phages) and the other 12 received a placebo. Clinical and microbiological indicators were measured at 7, 21 and 42 days after treatment. There were no reported side effects such as headache or dizziness, abdominal discomfort or pain, ear pain or rash and the phage-treated group reported statistically significant clinical improvements from baseline for all clinic days (erythema/inflammation, $\mathrm{p}=0.027$; ulceration/granulation/polyps, $\mathrm{p}=0.040$; discharge type, $\mathrm{p}=0.043$; odor, $\mathrm{p}=0.026)$ and significant reductions in $P$. aeruginosa numbers on days $21(\mathrm{p}=0.009)$ and $42(\mathrm{p}=0.016)$ that were not observed in the placebo group. Of note, although 44 patients were assessed for eligible inclusion in the trial, four were excluded because the swab-sampled $P$. aeruginosa was insensitive to the phages and 13 were excluded because mixed growth was observed on the initial swab. Matching the correct phage to the infectious agent is critical to the success of phage therapy and unpublished data from Georgian clinics suggest the critical need for sensitivity studies prior to treatment with phages [11]. For example, an FDA-approved Phase I trial examining the safety of a phage preparation for the treatment of venous leg ulcers containing phages against $P$. aeruginosa, $S$. aureus and $E$. coli reported no statistically significant differences between the treatment and control 
group in terms of the wound-healing frequency and rate [39]. This is not surprising given that the infectious agents were not tested for sensitivity to the phages prior to treatment. However, as no safety concerns were reported, the way is now clear for Phase II efficacy trials.

Unlike the previous smaller-scale trials, Phagoburn [40] is a European collaborative project that was launched in June 2013 to evaluate two phage cocktails for the treatment of burn wounds (PP1131 active against $P$. aeruginosa [13 phages in total] and PP0121 active against E. coli [12 phages in total]). The initial aim of the Phase I/II trial was to recruit 220 patients from intensive care units in 11 major burn facilities who would be randomly assigned to treatment and control groups [41]. In an article entitled 'Beleaguered phage therapy trial presses on,' Servick [42] outlined the setbacks that Phagoburn has encountered since its commencement. Firstly, the 12 months which had been allotted to establishing Good Manufacturing Practices for phage production took 20 months. Since the launch of the clinical phase of the trial in July 2015, only 15 eligible patients with $P$. aeruginosa infections and one with E. coli had been recruited. This can be explained by the fact that only patients presenting with a single bacterial infection are eligible but most are colonized with more than one type of bacterium. Consequently, the E. coli aspect of the study was terminated. Another setback that put patient recruitment on hold has been the request by France's National Agency for the Safety of Medicines and Health Products for proof of phage stability over time. Considering that the cocktail contains 13 highly similar phages, generating such data is extremely challenging. However, in June 2016, Servick [42] reported that recruitment had once again commenced since regulators accepted provisional measures demonstrating the sterility of the product along with its antibacterial activity. The company which developed the cocktail (Pherecydes Pharma, Romainville, France) is now investigating options to reduce the diversity of phages to decrease complexity, although such a move could reduce potency. Despite these issues, it is hoped that Phagoburn will successfully recruit 110 patients and it was hoped that efficacy data would be available by February or March 2017, but as of yet, no such data have been released. However, in March 2018, a notice on the Phagoburn website [40] stated that a detailed report showing results and outcomes will be added to the website shortly following scientific review.

E. coli

The species E. coli contains a diverse group of bacteria consisting of commensal and pathogenic members. They are generally found in the intestines of humans and animals where the commensals form an important component of the healthy intestinal microbiota [43]. Pathogenic members can cause childhood diarrhea, travelers' diarrhea in adults, bloody diarrhea, urinary tract infections, dysentery, meningitis, peritonitis, septicemia, hemolytic uremic syndrome [44,45] or even thrombotic thrombocytopenic purpura [46]. In 2009, it was reported that the appearance of antibiotic-resistant E. coli strains was rapidly rising, particularly in relation to fluoroquinolones and third-and fourth-generation cephalosporins [47]. In 2016, a plasmid-encoded colistin-resistance mechanism was identified in E. coli isolates from China [48] and the USA [49] designated Mcr-1. Until now, reported colistin resistance has been confined to chromosomal mutations, which tended to be unstable and imposed a fitness cost on the bacterial host. The transmissible nature of $m c r-1$ heralds the inevitable progression of Enterobacteriaceae from extensive drug resistance to pan-drug resistance [48].

Phages against $E$. coli have generally been isolated from sewage, hospital waste water, polluted rivers and human or animal fecal samples [50]. A few studies have investigated the safety of $E$. coli phages following oral administration to humans and to date no adverse effects have been reported [51-53].

The Nestle Research Centre, Switzerland and the International Centre for Diarrhoeal Diseases Research in Bangladesh have been in collaboration over the last 20 years to investigate the efficacy of alternative treatments for diarrheal diseases [44]. Recently, they performed a Phase II clinical trial using phages on 120 children suffering from acute diarrhea, who were infected with E. coli [54]. A detailed report of this trial from initial phage isolation right though to interpretation of results has been recently published [44]. Two phage preparations were under investigation, the Microgen phage cocktail targeting E. coli and Proteus infections, which had been previously analyzed by metagenomic analysis [53], and a T4-like phage-cocktail active against $E$. coli pathogens only (detailed isolation, characterization and production described in [44]). An initial safety test in patients was performed to ensure that the phages would not cause the release of large quantities of bacterial endo- and exotoxins in the gut upon lysis of potentially high titers of $E$. coli pathogens, which could cross into the bloodstream via a damaged leaky gut barrier and result in a Jarisch-Herxheimer reaction - an adverse reaction resulting from bacterial lysis due to antibiotic treatment. None of the patients showed toxic reactions [44]. In the Phase II trial, patients were randomized into three groups and received either the T4-phage cocktail $\left(3.6 \times 10^{8} \mathrm{pfu}\right)$, the Microgen product (1.4 
$\times 10^{9} \mathrm{pfu}$ ) or placebo (colored water), in addition to the standard care of oral rehydration solution combined with zinc treatment [54]. While both groups receiving phage treatments showed significant increases in fecal phage titres compared with placebo (with the Microgen group displaying the highest number of phages), phage therapy did not improve diarrhea outcome above the standard oral rehydration solution/zinc treatment. Subsequent microbiological analysis of patient stools suggested several reasons as to why phage therapy failed in this instance. First, low levels of pathogenic E. coli were detected; in fact, pathogen and commensal E. coli represented less than $5 \%$ of the total fecal bacterial population, suggesting that phage amplification in the intestine was not possible due to the low levels of target bacteria. Hence, the detected phages in the fecal samples represented treatment phages which passively transited the gut. Mixed infections were frequently observed in stools, despite the prescreening step to select patients infected only with the E. coli pathogen; thus, the causative agent of the diarrhea is unclear. Interestingly, microbiota analysis revealed a correlation between diarrhea and increased levels of Streptococcus. The abundance of these streptococci decreased as recovery from diarrhea ensued that coincided with a parallel increase in Bifidobacterium abundance, leaving the authors to speculate if Streptococcus is the causative agent of the observed diarrhea, a hypothesis also favored by Chinese researchers investigating the causative agent of diarrhea in hospitalized children for which no specific pathogen could be identified [55]. Further work is clearly warranted to fully understand the etiological agents responsible for childhood diarrhea and to appreciate phage-host interactions in the complex microbial ecosystem of the gut. However, it is possible that phage therapy may be more suited to indications where the causative agent can be easily identified and treatment ensures contact between phage and pathogen (e.g., skin and soft tissue infections and respiratory infections).

S. aureus

S. aureus is carried on the skin or mucous membranes of between 25 and $30 \%$ of healthy humans [56]. It is considered one of the most important human pathogens due to its ubiquitous occurrence and virulence potential [57]. S. aureus causes 11,000 deaths annually in the USA alone, accompanied by an annual cost of approximately $\$ 14$ billion [58] predominantly due to antibiotic-resistant strains [59]. Resistance to the antibiotics methicillin (and related antibiotics), the cephalosporins and vancomycin is of particular concern [60].

Phages against $S$. aureus have been isolated from various locations including sewage [61], farmyard slurry [62], the milk of a cow with mastitis [63] and skin [64] as examples. By 2014, it was reported that more than 200 lytic S. aureus phages had been characterized [65].

$S$. aureus phages have the advantage of being active against most members of the species owing to their relatively homogenous surface properties, unlike phages of Gram-negative organisms such as E. coli [66]. In this respect, $S$. aureus phage cocktails tend to be more commercially attractive due to the requirement for relatively few phages (2-3) [65]. The efficacy of $S$. aureus phages in disease treatment has been cited in several articles [11,25,65]. Most recently, topical administration with $S$. aureus phages (eye drops and nasal spray) and intravenous treatment successfully cleared a corneal abscess and interstitial keratitis in a 65-year old woman infected with vancomycinresistant $S$. aureus [22].

The phage-based biotechnology company Ampliphi Biosciences performed a Phase I clinical trial investigating the preliminary effectiveness and safety of a propriety $S$. aureus phage cocktail (AB-SA01) for the treatment of chronic rhinosinusitis. The results were presented at the 2017 Australian Society of Otolaryngology Head and Neck Surgery Meeting in April 2017. The cocktail was well tolerated with no reported safety issues. S. aureus numbers decreased in all nine treated patients and preliminary 3-month follow-up data revealed continued improvement of symptoms [67], leading the way for a Phase II clinical trial.

An essential consideration for phage therapy in the treatment of disease is the consequence of phage interactions with the host immune response. Humans are exposed to phages daily, through the environment and diet [68] and it is now known that phages are a major component of the intestinal microbiota [69]. In addition, a number of studies suggest that phages can penetrate other regions of the body and have been detected in the blood of healthy donors [70], in the pericardial fluid of patients suffering from pericarditis [71] as well as in the blood and urinary tract of patients following phage therapy [72]. Thus, knowledge regarding the host immune response to phages should be considered when administering phage therapy [73]. Indeed, by investigating the antiphage activity of phagocytes, antibodies and serum complement in mice and incorporating the results into a mathematical model, Hodyra-Stefaniak et al. revealed that the mammalian host immune response to bacterial infection simultaneously removes phages [74]. The group suggests that when considering phage therapy, 'the complexity of mammalian immunity and the mammalian host-versus-phage (MHvP) immune response' should be considered. However, results from human patients suggest 
that phage therapy can still be efficacious in the event of immune induction. In a study involving 122 patients attending the Phage Therapy Unit in Poland, Eusiak-Szelachowska et al. reported that induction of antiphage antibodies to phage therapy in patient sera depended on route of phage administration whereby orally treated patients had low antiphage activity compared with some of those treated locally [75]. Importantly, the authors state that induction of antiphage activity in patient sera during or after treatment did not exclude a favorable outcome of treatment. Likewise, a study of 20 patients receiving the staphylococcal MS-1 phage cocktail orally and/locally reported that most did not reveal higher levels of antiphage antibodies in their sera during treatment [76]. Moreover, efficacy of phage treatment was not compromised in patients who revealed an increased immune response (mostly $\operatorname{IgG}$ and $\operatorname{IgM}$ ). Interestingly, however, some patients who exhibited weak antiphage antibody induction before and during treatment failed to respond to treatment. Thus, the authors concluded that antiphage antibody induction does not necessarily influence the outcome of phage therapy. More recently, phage neutralization levels in the sera could not be correlated with the outcome of phage therapy in a study involving 62 patients and 30 healthy volunteers [77]. A study investigating the effect of phage preparations on the bacterial killing activity of phagocytes in 51 patients receiving phage treatment for chronic bacterial infections revealed that phages do not interfere with phagocyte activity [78]. While these studies provide further support for the safety of phage therapy and suggest that the immune response does not necessarily interfere with the efficacy of treatment, more studies of this nature are vital and may even shed light on the often conflicting results observed for phage therapy treatments (Table 1) and pave the way for innovative formulation and delivery strategies. As an example, liposome-entrapped phages were $100 \%$ protected from murine-neutralizing antibodies, whereas unentrapped phages were neutralized within $3 \mathrm{~h}$ of antibody exposure [79], albeit neutralization does not necessarily affect the outcome of phage treatment in humans $[75,77]$. Moreover, the entrapped phages were successfully delivered into murine macrophages, resulting in 94.6\% killing of Klebsiella pneumoniae compared with $21.4 \%$ killing by the unentrapped phages after $24 \mathrm{~h}$. In conjunction with amikacin, the entrapped phages were capable of eradicating mature $K$. pneumoniae biofilm. This suggests that phage formulation could be a critical component in determining the success rate of clinical outcomes. Thus, pharmacokinetic and pharmacodynamic studies of phage preparations and protected phage formulations should be essential prerequisites toward determining the efficacy of phage therapy in clinical applications.

\section{Is phage resistance an issue?}

It is known that bacteria harbor an array of phage-resistance mechanisms with the ability to impede the phage lytic lifecycle at various stages [80]. Adsorption inhibition describes a mechanism whereby the bacterium prevents phage attachment to the receptor resulting in the generation of bacteriophage insensitive mutants (BIMs) and can be achieved by multiple mechanisms including modification of the receptor through mutations, loss of the receptor (e.g., reduced expression) or production of extracellular polysaccharides, which shield the receptor. Restriction modification systems generally consist of a restriction endonuclease, which cleaves foreign DNA at specific recognition sites. Host DNA is protected through modification by a partner methyltransferase that recognizes the same sites. The CRISPR-Cas system has been described as adaptive immunity for prokaryotes and cleaves invading DNA that contains a nucleotide sequence matching a cognate sequence (spacer) in the host's CRISPR array, the spacer having been incorporated into the CRISPR array during a previous exposure [81]. Abortive infection describes a diverse range of host mechanisms, which can interfere with different stages of the phage infection process from phage transcription right through to phage packaging [82] and generally results in death of the infected cell. Recently, a novel phage resistance system, termed BREX (bacteriophage exclusion) was identified [83], and is distantly related to the phage growth limitation system of Streptomyces coelicolor [84].

Unlike antibiotics phages have the ability to evolve and escape these mechanisms [80]. However, this generally gives way to an escalation of defense and counter-defense cycles as bacteria evolve against the 'co-evolved' phages and so on and is termed the arms race dynamic. On an optimistic note, the acquisition of phage resistance may result in reduced fitness in the bacterial cell with deleterious consequences for growth rate and even pathogenicity particularly if the phage receptor is required for virulence, a topic which has been recently reviewed by León and Bastías [85]. For example, while $P$. aeruginosa phage-resistant derivatives appeared following a single dose of phages to fibrin clots in vitro, they failed to emerge in vivo in a rat model of endocarditis due to $P$. aeruginosa following a single dose of the same phage cocktail [86]. The authors attribute this to potential phage resistance mutations in important bacterial surface determinants required for infectivity. Because of this phenomenon, high-throughput screens are now being used to identify small-effector molecules that inhibit one or more steps involved in the production of a functioning phage receptor in the search for next-generation therapeutics [87]. Moreover, phage 
resistance has also been associated with increases in antibiotic susceptibility. This was recently observed in a $P$. aeruginosa phage-resistant mutant to phage OMKO1, which utilizes the outer membrane porin (OprM) of the multidrug efflux systems MexAB and MexXY as a receptor [88]. In this case, phage resistance altered the efflux pump mechanism of the multidrug-resistant $P$. aeruginosa resulting in increased sensitivity to drugs from several antibiotic classes.

The phage cocktail has been touted as an effective solution whereby two or more phages infect the same target bacterium, thus reducing the likelihood of emerging resistance. For example, a cocktail of $C$. difficile (temperate) phages resulted in the complete lysis of the pathogen in vitro while a single phage caused an initial reduction followed by the emergence of resistant mutants, albeit these resistant colonies were susceptible to infection by an unrelated phage [89]. In the same study, the optimized phage combinations delayed the onset of symptoms of C. difficile infection by $33 \mathrm{~h}$ in a hamster model when compared with the control but failed to elicit complete protection. In another study, a single phage proved as effective as a phage cocktail at reducing Campylobacter jejuni load in commercial broilers, although the cocktail delayed the emergence of phage resistance [90]. Pretreatment of hydrogel-coated catheters with $P$. aeruginosa phage M4 significantly reduced biofilm formation by $P$. aeruginosa M4 within $24 \mathrm{~h}$ when compared with the untreated control (2.8 log reduction) [91]. Biofilm isolates resistant to phage M4 were isolated and their phage susceptibility profiles were used as a guide to generate a five-phage cocktail that generated a significant reduction in biofilm formation when compared with treatment with the single phage. However, five distinct $P$. aeruginosa morphologies were isolated from the cocktail-treated catheters and while four remained sensitive to at least one of the phages in the cocktail, one isolate was resistant to all five phages, and demonstrated greater biofilm formation capacity than the M4 parent strain. The authors suggest that continued screening might result in the identification of a lytic phage against the resistant isolate but that the phage cocktail approach is best suited to applications that provide protection against initial bacterial challenge rather than those that require prolonged protection. O' Flynn et al. demonstrated that BIMs emerged at the same frequency against two phages in a three-phage cocktail $\left(10^{-6}\right.$ colony-forming units [CFU]), while the frequency of BIM emergence against the third phage in the cocktail was higher, at $10^{-4} \mathrm{CFU}$ [92]. However, the BIMs exhibited an altered morphology when compared with the parent strain, being coccoid rather than rod-shaped, and reverted to phage sensitivity after 50 generations in the absence of phages.

These studies suggest that while the cocktail at most delays the emergence of phage-resistant mutants, it does not eliminate the risk. However, the phage cocktail was never intended to be a static, predefined treatment but one which can be adapted as needed as is practiced in Georgia and Poland. It is also important to point out that good results have been reported for phage monotherapy (the use of a single phage) in human studies [93], albeit it has been reported that phage resistance did occur during the course of treatments [26]. Despite this, Górski et al. state that preliminary clinical observations have not indicated that the phage cocktail is superior to the single phage [93].

The ubiquitous nature of phages suggests that alternative lytic phages should be easily isolated. To test this hypothesis, Mattila et al. investigated if on-demand phage isolation could be achieved against the most common hospital-borne-resistant pathogens including MRSA, extended spectrum $\beta$-lactamase E. coli and $K$. pneumoniae, multidrug-resistant $P$. aeruginosa, vancomycin-resistant Enterococcus, Acinetobacter baumanii and a variety of Salmonella species using sewage water as a source [94]. The study involved 283 isolation attempts against 83 different host strains. On-demand phage isolation was feasible for $P$. aeruginosa, Salmonella and extended spectrum $\beta$-lactamase E. coli and $K$. pneumoniae. The probability of finding phages against Enterococcus and A. baumanii was less than $40 \%$, while finding phages against MRSA proved very difficult. However, as previously mentioned, phages against $S$. aureus tend to have a broader host range [66]. For example, phage $\mathrm{K}$ was shown to lyse 22 of 36 distinct human-derived MRSA strains, as well as teicoplanin- and vancomycin-resistant strains and eight other tested species, namely S. epidermidis, S. saprophyticus, S. chromogenes, S. capilis, S. hominis, S. heamolyticus, S. caprae and S. hyicus [95]. Interestingly, the phage was initially deemed incapable of infecting 14 human-derived MRSA strains; however, incubation of the phage with these strains resulted in modified phage variants which were capable of lysing the previously sensitive strains. This process of phage 'training' generally involves serial transfers of the phage in its ancestral host. It has resulted in the generation of an improved phage in terms of infectivity against $P$. aeruginosa cystic fibrosis isolates, since fewer $P$. aeruginosa strains (50\% less) could evolve resistance against the evolved phage compared with the ancestral phage [96] and in generating a $P$. aeruginosa phage with improved in vivo efficacy and expanded host range [97]. In this study, the improved phage was fully sequenced revealing a difference of two single nucleotide mutations when compared with the parent phage highlighting the ease with which phages can be optimized over conventional antibiotics. 
Moreover, by coincubating four phages with multiple $P$. aeruginosa strains, Mapes et al. generated undefined phage mixtures with expanded host range which were used to generate defined cocktails with predictable and broad host ranges and which were capable of reducing biofilm formation and reducing preexisting biofilms when used against sensitive isolates [98]. Indeed, it has been suggested that multiple strains should be used during phage isolation to produce broader host range phages [99]. Specifically, phages isolated against $E$. faecalis using a mixture of two host strains exhibited broader host ranges than phages isolated using a single host strain.

Overall, these studies reveal that phage cocktails or indeed single phage in the case of monotherapy can be upgraded by either isolating new lytic phages or improving the infectivity and host range of existing phages. Interestingly, in a 'step-by-step' approach, Gu et al. generated a phage cocktail against $K$. pneumoniae using wildtype bacteria to isolate the initial lytic phage and then its phage-resistant derivatives were used to isolate further lytic phages and this process was continued until the last phage-resistant bacterium was sensitive to the next isolated phage resulting in a cocktail targeting the wild-type strain and its phage-resistant variants [100]. The cocktail generated in this way was capable of protecting four out of five mice from $K$. pneumoniae bacteremia.

While often the killing potential of a cocktail is greater than the combined killing potential of the single phage - referred to as synergy [14], it is important to note that phages in a cocktail may also interfere with each other - and this was observed in food trials using E. coli phages CEV1 and CEV2, whereby CEV1 prevented CEV2 reproduction in E. coli cells [101]. In this regard, it is critical that phages within a cocktail are compatible. Indeed, Gill and Hyman advise using phages which adsorb to independent bacterial receptors [102].

Overall, phage resistance can be much more easily overcome than antibiotic resistance using updated, targeted cocktails or an alternative lytic phage in the case of monotherapy; however, this will depend on the regulatory framework governing phage use for medical purposes. In this regard, the 'biological master file' approach recommended by Fauconnier [30] seems the most fitting course of action by ensuring that the most effective phage or phage combinations can be used at the most appropriate times for disease treatment.

\section{Conclusion}

This is an exciting juncture in phage biology, where we can look back on the knowledge gleaned over the last 100 years, understand the successes and failures, and hopefully move phage therapy forwards with realistic expectations - a viable strategy to counter the antibiotic resistance crisis of the 21 st century, giving us access to targeted antimicrobials to treat infections but which do not damage the microbial partners which are key to the health of the human. Inarguably, one of the greatest impediments to the success of phage therapy in parts of the world other than eastern Europe is current legislation. The conundrum is that the legislation is unlikely to change if clinical trials fail to convince the regulators that phage therapy works, yet it appears that the legislation is limiting the very potential of this therapy in current clinical trials. Regulatory authorities must understand the dynamic nature of phage therapy if it is to be a success. Thus, the support of regulatory authorities along with consumer and practitioner education and acceptance will be critical. Furthermore, it is important that scientists conduct rigorous trials to the highest standards to convince regulatory authorities that phages present a viable alternative or addition to existing therapies. It is important that we learn from the mistakes of the past, and perhaps we may even have to limit the many possible applications of phages to properly exploit their potential in human health. Should the phages we plan to use in human medicine also be used in food, the environment and agriculture? Can phages be used systemically or are they limited to oral and topical applications? We stand at the brink of an explosion of interest in phages as therapeutics, as diagnostic tools and as precision tools for shaping and understanding the microbiome - truly it seems as if we may be entering the next age of the phage.

\section{Future perspective}

Perhaps one of the most vital keys in unleashing the full potential of phage therapy is the ability to rapidly identify the pathogen(s) of concern in the first place. Rapid and accurate identification should ensure that the most accurate phage 'prescription' is applied. To this end, phages themselves are proving to be powerful biosensing tools with the ability to discern microbes to the strain level whether through phage amplification and phage lysis assays, or as reporter phages and phage-based bionsensors, a topic which has been reviewed by Tawil et al. [103]. These assays generally enable the rapid detection of low numbers of bacterial cells without the need for preenrichment steps. As an example, the Enzyme-linked Long Tail Fibre Assay recently developed by Denyes et al. has the capacity to

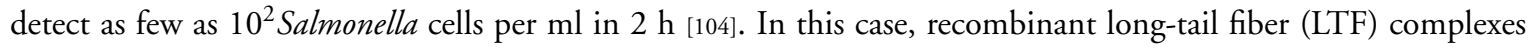
from phage S16 were conjugated with horse radish peroxidase-LTF and another set of recombinant tail fiber 
complexes were used to coat paramagnetic beads (LTF-MBs). The LTF-MBs were capable of capturing Salmonella from various foods enabling detection of 1-10 CFU per $25 \mathrm{~g} / \mathrm{ml}$, while the horse radish peroxidase-LTF allowed rapid colorimetric detection of bead-captured Salmonella. Continued research into this field is essential for the development of low-cost, easy-to-use assays with the capacity for pathogen identification in the shortest time possible.

Genetic engineering also has the potential to generate superior phages with broader host ranges, better survival rates, and could help to convert lysogenic phages to obligately lytic lifecycles [105]. However, the use of genetically modified phages for clinical purposes would undoubtedly be met with resistance given the level of opposition to genetic engineering. A recent expert round table on the acceptance and reimplementation of phage therapy [106] suggests there is sufficient potential in already existing phages to create new opportunities for treating bacterial infections. For example, combining phages with antibiotics has generated impressive synergistic results [86,107] warranting further studies of this nature and suggests that combining phages with other antimicrobials is worth investigating.

Moreover, in the introduction we stated that $N$. gonorrhoeae was resistant to all known antibiotics in certain countries. While lytic phages against this pathogen have not been isolated to date, its genome sequence has been shown to harbor multiple intact or truncated prophages [108]. One such prophage, Ngo $\Phi 6$, has recently been used in the development of a gonococcal vaccine and resulted in the generation of antibacterial sera against $N$. gonorrhoeae following oral immunization of rabbits with attenuated Salmonella cells expressing the $N$. gonorrhoeae filamentous phage [109].

In this regard, continued research into the mammalian immune response to phages is essential and should add to our understanding of clinical trial outcomes and as in the study above may well lead to the development of novel vaccine treatments. Research has shown that phages are capable of inducing an immune response though it does not appear to influence treatment outcomes [75,77]. Despite this, it is possible that phages could benefit from protection such as encapsulation. We had previously speculated that the inhibition of phage infection in raw milk was due to complement (causing clumping of the target pathogen) [110], which might suggest that applications in blood could be limited. Such formulations should ensure longer phage survival in the mammalian blood system or aid survival in the harsh gastrointestinal conditions. Moreover, recent studies suggest that phages may have an important role to play in immune homeostasis [111], with particular emphasis on the gut [112] where phages have been shown to account for approximately $90 \%$ of gut virome (viruses and phages) composition [113]. In addition, a recent study revealed that intraperitoneal application of phage lysates to a murine model of rheumatoid arthritis did not aggravate the course of the condition but rather the purified phage lysate exerted an immunosuppressive effect [114]. While further research is warranted, such results suggest potential for the development of new and innovative phage-based therapeutics for clinical use.

Financial \& competing interests disclosure

This publication has been supported by a grant from Science Foundation Ireland (SFI; www.sfi.ie) under grant number $\mathrm{SFI} / 12 / \mathrm{RC} / 2273$. The authors have no other relevant affiliations or financial involvement with any organization or entity with a financial interest in or financial conflict with the subject matter or materials discussed in the manuscript apart from those disclosed.

No writing assistance was utilized in the production of this manuscript.

\section{Open access}

This work is licensed under the Attribution-Non Commercial-No Derivatives 4.0 Unported License. To view a copy of this license, visit http://creativecommons.org/licenses/by-nc-nd/4.0/ 
Executive summary

The status of phage therapy in eastern Europe \& the western world

- Phage therapy continues to be used in certain regions of the world including eastern Europe for both prophylactic and therapeutic purposes. However, the paucity of controlled clinical trials makes it difficult to evaluate its effectiveness by western standards.

- Current EU regulatory frameworks dramatically limit the potential of phage therapy given that its success is dependent on the ability to update phage preparations or develop personalized preparations with pathogen-specific phages.

- A novel regulatory framework to address the issue of customized phage therapy medicinal products is required.

Phages \& infectious disease

- Three human pathogens, Pseudomonas aeruginosa, Escherichia coli and Staphylococcus aureus, are the focus of recent and ongoing clinical trials.

- It is critical that the correct phage is matched to the infectious agent prior to treatment.

- The consequence of phage interactions with the host immune response is an important consideration for phage therapy. Pharmacokinetic and pharmacodynamic studies of phage preparations are essential toward determining the efficacy of phage therapy in clinical applications.

Is phage resistance an issue?

- The phage cocktail has been touted as the solution to phage resistance. However, at most the phage cocktail will delay the emergence of phage-resistant mutants, but will not eliminate the risk as a standalone treatment. The success of the phage cocktail, or a single-phage treatment, is dependent on the ability to be able to adapt it as needed with pathogen-specific phage(s).

- New phages can be easily isolated from the environment or by 'training' existing phages to generate improved phages in terms of infectivity and host range. However, current regulations make it virtually impossible to take advantage of this key feature of phage therapy.

\section{References}

Papers of special note have been highlighted as: $\bullet$ of interest; $\bullet \bullet$ of considerable interest

1. Wittebole X, De Roock S, Opal SM. A historical overview of bacteriophage therapy as an alternative to antibiotics for the treatment of bacterial pathogens. Virulence 5(1), 226-235 (2014).

2. Spellberg B, Taylor-Blake B. On the exoneration of Dr. William H. Stewart: debunking an urban legend. Infect. Dis. Poverty 2, 3 (2013).

3. World Health Organisation. Antibiotic resistant gonorrhoea on the rise, new drugs needed. (2017). www.who.int/mediacentre/news/releases/2017/Antibiotic-resistant-gonorrhoea/en/

4. Centers for Disease Control and Prevention (CDC). Antibiotic/antimicrobial resistance. (2016). www.cdc.gov/drugresistance/

5. European Medicines Agency (EMA) and European Centre for Disease Prevention and Control (ECDC). The bacterial challenge: time to react a call to narrow the gap between multidrug-resistant bacteria in the EU and development of new antibacterial agents. EMA and ECDC, Stockholm, Sweden (2009).

http://ecdc.europa.eu/en/publications/publications/0909_ter_the_bacterial_challenge_time_to_react.pdf

6. Ferrer M, Méndez-García C, Rojo D, Barbas C, Moya A. Antibiotic use and microbiome function. Biochem. Pharmacol. 134, 114-126 (2016).

7. Ianiro G, Tilg H, Gasbarrini A. Antibiotics as deep modulators of gut microbiota: between good and evil. Gut 65(11), 1906-1915 (2016).

8. Theriot CM, Young VB. Microbial and metabolic interactions between the gastrointestinal tract and Clostridium difficile infection. Gut Microbes 5(1), 86-95 (2014).

9. Salmond GPC, Fineran PC. A century of the phage: past, present and future. Nat. Rev. Microbiol. 13(12), 777-786 (2015).

10. Hershey AD, Chase M. Independent functions of viral protein and nucleic acid in growth of bacteriophage. J. Gen. Physiol. 36(1), 39-56 (1952).

11. Kutter E, De Vos D, Gvasalia G et al. Phage therapy in clinical practice: Treatment of human infections. Curr. Pharm. Biotechnol. 11(1), 69-86 (2010).

12. Kutateladze M. Experience of the Eliava Institute in bacteriophage therapy. Virol. Sin. 30(1), 80-81 (2015).

13. Zschach $\mathrm{H}$, Joensen KG, Lindhard B et al. What can we learn from a metagenomic analaysis of a Georgian bacteriophage cocktail? Viruses 7(12), 6570-6589 (2015).

14. Weber-Dąbrowska B, Jończyk-Matysiak E, Zaczek M et al. Bacteriophage procurement for therapeutic purposes. Front. Microbiol. 7 , 1177 (2016).

15. Phage Therapy Unit of the Medical Centre of the Institute of Immunology and Experimental Therapy PAS. www.iitd.pan.wroc.pl/en/OTF/ 
16. Markoishvili K, Tsitlanadze G, Katsarava R, Morris JG Jr, Sulakvelidze A. A novel sustained-release matrix based on biodegradable poly(ester amide)s and impregnated with bacteriophages and an antibiotic shows promise in management of infected venous stasis ulcers and other poorly healing wounds. Int. J. Dermatol. 41, 453-458 (2002).

17. Weber-Dąbrowska B, Mulczyk M, Górski A. Bacteriophages as an efficient therapy for antibiotic-resistant septicaemia in man. Transplant. Proc. 35, 1385-1386 (2003).

18. Jikia D, Chkhaidze N, Imedashvili E et al. The use of a novel biodegradable preparation capable of the sustained release of bacteriophages and ciproflaxicin, in the complex treatment of multidrug-resistant Staphylococcus aureus-infected local radiation injuries caused by exposure to Sr90. Clin. Exp. Dermatol. 30(1), 23-26 (2005).

19. Leszczyński P, Weber-Dąbrowska B, Kohutnicka M, Łuczak M, Górecki A, Górski A. Successful eradication of methicillin-resistant Staphylococcus aureus (MRSA) intestinal carrier status in a healthcare worker - case report. Folia Microbiol. (Praha) 51(3), 236-238 (2006).

20. Międzybrodzki R, Fortuna W, Weber-Dąbrowska B, Górski A. Phage therapy of staphylococcal infections (including MRSA) may be less expensive than antibiotic treatment. Postepy Hig. Med. Dosw. 61, 461-465 (2007).

21. Letkiewicz S, Międzybrodzki R, Fortuna W, Weber-Dąbrowska B, Górski A. Eradication of Enterococcus faecalis by phage therapy in chronic bacterial prostatitis case report. Folia Microbiol. (Praha) 54(5), 457-461 (2009).

22. Fadlallah A, Chelala E, Legeais J-M. Corneal infection therapy with topical bacteriophage administration. Open Ophthalmol. J. 9, 167-168 (2015).

23. Schooley RT, Biswas W, Gill JJ et al. Development and use of personalized bacteriophage-based therapeutic cocktails to treat a patient with a disseminated resistant Acinetobacter baumannii infection. Antimicrob. Agents Chemother. 61(10), pii:e00954-17 (2017).

24. Jennes A, Merabishvili M, Soentjen P et al. Use of bacteriophages in the treatment of colistin-only-sensitive Pseudomonas aeruginosa septicaemia in a patient with acute kidney injury - a case report. Crit. Care 21, 129 (2017).

25. Abedon ST, Kuhl SJ, Blasdel BG, Kutter EM. Phage treatment of human infections. Bacteriophage 1(2), 66-85 (2011).

26. Międzybrodzki R, Borysowski J, Weber-Dąbrowska B et al. Clinical aspects of phage therapy. Adv. Virus Res. 83, 73-121 (2012).

27. European Medicines Agency (EMA). Workshop on the therapeutic use of bacteriophages. (2015). www.ema.europa.eu/ema/index.jsp?cu rl=pages/news_and_events/events/2015/05/event_detail_001155.jsp\&mid=WC0b01ac058004d5c3

28. European Commission. Directive 2001/83/EC of the European Parliament and of the council of 6 November 2001 on the community code relating to medicinal products for human use (consolidated version: 16/11/2012). In: EudraLex - The Rules Governing Medicinal Products in the European Union (Volume 1), Pharmaceutical Legislation: Medicinal Products For Human Use. http://ec.europa.eu/health/files/eudralex/vol-1/dir_2001_83_consol_2012/dir_2001_83_cons_2012_en.pdf

29. Pelfrene E, Willebrand E, Sanches AC, Sebris Z, Cavaleri M. Bacteriophage therapy: a regulatory perspective. J. Antimicrob. Chemother. 71(8), 2071-2074 (2016)

30. Fauconnier A. Regulating phage therapy: the biological master file concept could help to overcome regulatory challenge of personalized medicines. EMBO Rep. 18(2), 198-200 (2017).

- Proposes the 'biological master file' concept and addresses the issues with current legislation in relation to phage therapy.

31. Kingwell K. Bacteriophage therapies re-enter clinical trials. Nat. Rev. Drug Discov. 14, 515-516 (2015).

32. Parracho HM, Burrowes BH, Enright MC, McConville ML, Harper DR. The role of regulated clinical trials in the development of bacteriophage therapeutics. J. Mol. Genet. Med. 6, 279-286 (2012).

33. Danis-Wlodarczyk K, Vandenheuvel D, Bin Jang H et al. A proposed integrated approach for the preclinical evaluation of phage therapy in Pseudomonas infections. Sci. Rep. 6, 28115 (2016).

34. Lister PD, Wolter D, Hanson ND. Antibacterial-resistant Pseudomonas aeruginosa: clinical impact and complex regulation of chromosomally encoded resistance mechanisms. Clin. Microbiol. Rev. 22(4), 582-610 (2009).

35. Hraiech S, Brégeon F, Rolain JM. Bacteriophage-based therapy in cystic fibrosis-associated Pseudomonas aeruginosa infections: rationale and current status. Drug Des. Dev. Ther. 9, 3653-3663 (2015).

36. Ceyssens PJ, Noben JP, Ackermann HW et al. Survey of Pseudomonas aeruginosa and its phages: de novo peptide sequencing as a novel tool to assess the diversity of worldwide collected viruses. Environ. Microbiol. 11(5), 1303-1313 (2009).

37. Alemayehu D, Casey PG, McAuliffe $\mathrm{O}$ et al. Bacteriophages $\varphi \mathrm{MR} 299-2$ and $\varphi \mathrm{NH}-4$ can eliminate Pseudmomas aeruginosa in the murine lung and on cystic fibrosis lung airway cells. MBio 3(2), e00029-12 (2012).

38. Wright A, Hawkins CH, Änggård EE, Harper DR. A controlled clinical trial of a therapeutic bacteriophage preparation in chronic otitis due to antibiotic resistant Pseudomonas aeruginosa; a preliminary report of efficacy. Clin. Otolaryngol. 34(4), 349-357 (2009).

39. Rhoads DD, Wolcott RD, Kuskowski MA, Wolcott BM, Ward LS, Sulakvelidze A. Bacteriophage therapy of venous leg ulcers in humans: results of a Phase I safety trial. J. Wound Care. 18(6), 237-243 (2009).

40. Phagoburn. www.phagoburn.eu

41. Sansom C. Phage therapy for severe infections tested in the first multicentre trial. Lancet 15(12), 1384-1385 (2015).

42. Servick K. Beleaguered phage therapy trial presses on. Science 352(6293), 1506 (2016). 
- Details the setbacks and obstacles associated with a phage therapy clinical trial.

43. Centres for Disease Control and Prevention. Escherichia coli. (2015). www.cdc.gov/ecoli/general/index.html

44. Sarker SA, Brüssow H. From bench to bed and back again: phage therapy of childhood Escherichia coli diarrhoea. Annal. NY Acad. Sci. 1372(1), 42-52 (2016).

-• Detailed account of phage isolation and preparation procedures from testing in mice to safety tests in humans in a Phase I clinical trial. The study then describes the Phase II clinical trial, which failed to alleviate diarrhea in children but provides in-depth discussion on the outcome and possible reasons for the lack of success.

45. Tadesse DA, Zhao S, Tong E et al. Antimicrobial drug resistance in Escherichia coli from humans and food animals, United States, 1950-2002. Emerg. Infect. Diseases 18(5), 741-749 (2012).

46. Kovacs MJ, Roddy J, Grégoire S, Cameron W, Eidus L, Drouin J. Thrombotic thrombocytopenic purpura following hemorrhagic colitis due to Escherichia coli 0157:H7. Am. J. Med. 88(2), 177-179 (1990).

47. Collignon P. Resistant Escherichia coli - we are what we eat. Clin. Infect. Dis. 49(2), 202-204 (2009).

48. Liu YY, Wang Y, Walsh TR et al. Emergence of plasmid-mediated colistin resistance mechanism MCR-1 in animals and human beings in China: a microbiological and molecular biological study. Lancet 16(2), 161-168 (2016).

49. McGann P, Snesrud E, Maybank R et al. Escherichia coli harboring $m c r-1$ and blaCTX-M on a novel IncF Plasmid: first report of $m c r-1$ in the United States. Antimicrob. Agents Chemother. 60(7), 4420-4421 (2016).

50. Brüssow H. Phage therapy: the Escherichia coli experience. Microbiology 151(7), 2133-2140 (2005).

51. Bruttin A, Brüssow H. Human volunteers receiving Escherichia coli phage T4 orally: a safety test of phage therapy. Antimicrob. Agents Chemother. 49(7), 2874-2878 (2005).

52. Sarker SA, McCallin S, Barretto C et al. Oral T4-like phage cocktail application to healthy adult volunteers from Bangladesh. Virology 434(2), 222-232 (2012).

53. McCallin S, Sarker SA, Barretto C et al. Safety analysis of a Russian phage cocktail: from metagenomic analysis to oral application in healthy human subjects. Virology 443(2), 187-196 (2013).

54. Sarker SA, Sultana $S$, Reuteler G et al. Oral phage therapy of acute bacterial diarrhea with two coliphage preparations: a randomized trial in children from Bangladesh. EBio Med. 4, 124-137 (2016).

55. Jin D, Chen C, Li L et al. Dynamics of fecal microbial communities in children with diarrhea of unknown etiology and genomic analysis of associated Streptococcus lutetiensis. BMC Microbiol. 13, e141 (2013).

56. Wertheim HF, Melles DC, Vos MC et al. The role of nasal carriage in Staphylococcus aureus infections. Lancet Infect. Dis. 5(12), 751-762 (2005).

57. Grundmann H, Aanensen DM, van den Wijngaard CC et al. Geographic distribution of Staphylococcus aureus causing invasive infections in Europe: a molecular-epidemiological analysis. PLOS Med. 7(1) e1000215 (2010).

58. Klein E, Smith DL, Laxminarayan R. Hospitalizations and deaths caused by methicillin-resistant Staphylococcus aureus, United States, 1999-2005. Emerg. Infect. Dis. 13(12),1840-1846 (2007).

59. Vuong C, Yeh AJ, Cheung GYC, Otto M. Investigational drugs to treat methicillin-resistant Staphylococcus aureus. Expert Opin. Investig. Drugs 25(1), 73-93 (2016).

60. Centre for Disease Control and Prevention (CDC). Antibiotic resistance threats in the United States. (2013). www.cdc.gov/drugresistance/pdf/ar-threats-2013-508.pdf

61. Wang Z, Zheng P, Ji W et al. SLPW: A virulent bacteriophage targeting methicillin sesistant Staphylococcus aureus in vitro and in vivo. Front. Microbiol. 7, 934 (2016).

62. O’ Flaherty S, Ross RP, Flynn J, Meaney WJ, Fitzgerald GF, Coffey A. Isolation and characterization of two anti-staphylococcal bacteriophages specific for pathogenic Staphylococcus aureus associated with bovine infections. Lett. Appl. Microbiol. 41(6), 482-486 (2005).

63. Kwiatek M, Parasion S, Mizak L, Gryko R, Bartoszcze M, Kocik J. Characterization of a bacteriophage, isolated from a cow with mastitis, that is lytic against Staphylococcus aureus strains. Arch. Virol. 157(2), 225-234 (2012).

64. Chang Y, Shin H, Lee JH, Park CJ, Paik SY, Ryu S. Isolation and genome characterization of the virulent Staphylococcus aureus bacteriophage SA97. Viruses 7(10), 5225-5242 (2015).

65. Kaźmierczak Z, Górski A, Dąbrowska K. Facing antibiotic resistance: Staphylococcus aureus phages as a medical tool. Viruses 6(7), 2551-2570 (2014)

66. Kutter EM, Kuhl SJ, Abedon ST. Re-establishing a place for phage therapy in western medicine. Future Microbiol. 10(5), 685-688 (2015).

67. Ooi M. A Phase I clinical trial to evaluate the safety, tolerability and preliminary effectiveness of AB-SA01 in patients with chronic rhinosinusitis associated with Staphylococcus aureus infection. Presented at: The 2017 Australian Society of Otolaryngology Head and Neck Surgery Meeting, Adelaide, Australia, 23-26 March 2017.

68. Clokie MRJ, Millard AD, Letarov AV, Heaphy S. Phages in nature. Bacteriophage 1(1), 31-45 (2012). 
69. Manrique P, Bolduc B, Walk ST, van der Oost J, de Vos WM, Young MJ. Healthy human gut phageome. Proc. Natl Acad. Sci. USA 113(37), 10400-10405 (2016).

70. Breitbart M, Rohwer F. Method for discovering novel DNA viruses in blood using viral particle selection and shotgun sequencing. Biotechniques 39, 729-736 (2005).

71. Fancello L, Monteil S, Popgeorgiev N et al. Viral communities associated with human pericardial fluids in idiopathic pericarditis. PLoS ONE 9(4), e93367 (2014).

72. Weber-Dąbrowska B, Dabrowski M, Slopek S. Studies on bacteriophage penetration in patients subjected to phage therapy. Arch. Immunol. Ther. Exp. 35, 563-568 (1987).

73. Górski A, Międzybrodzki R, Borysowski J et al. Phage as a modulator of immune responses: practical implications for phage therapy. Adv. Virus Res. 83, 41-74 (2012).

74. Hodyra-Stefaniak K, Miernikiewicz P, Drapała J et al. Mammalian host-versus-phage immune response determine phage fate in vivo. Sci. Rep. 5, 14802 (2015).

-. A mathematical model was developed to interpret the impact of innate and adaptive immunity on phage pharmacokinetics, which demonstrated an indirect pathway of phage inhibition by bacteria that proceed through bacterial stimulation of the innate immune response of which the consequence is phage inhibition.

75. Eusiak-Szelachowska M, Zaczek M, Weber-Dąbrowska B et al. Phage neutralization by sera of patients receiving phage therapy. Viral Immunol. 27(6), 295-304 (2014).

76. Zaczek M, Łusiak-Szelachowska M, Jończyk-Matysiak E et al. Antibody production in response to staphylococcal MS-1 phage cocktail in patients undergoing phage therapy. Front. Microbiol. 7, 1681 (2016).

77. Łusiak-Szelachowska M, Zaczek M, Weber-Dąbrowska B et al. Antiphage activity of sera during phage therapy in relation to its outcome. Future Microbiol. 12(2), 109-117 (2017).

78. Jończyk-Matysiak E, Łusiak-Szelachowska M, Ktak M et al. The effect of bacteriophage preparations on intracellular killing of bacteria by phagocytes. J. Immunol. Res. 2015, 482863 (2015).

79. Singla S, Harjai K, Katare O, Chhibber S. Encapsulation of bacteriophage in liposome accentuates its entry in to macrophage and shields it from neutralizing antibodies. PLoS ONE 11(4), e0153777 (2016).

80. Samson JE, Magadán AH, Sabri M, Moineau S. Revenge of the phages: defeating bacterial defences. Nat. Rev. Microbiol. 11(10), 675-687 (2013).

81. Mohanraju P, Makarova KS, Zetsche B, Zhang F, Koonin EV, van der Oost J. Diverse evolutionary roots and mechanistic variations of the CRISPR-Cas systems. Science 353(6299), aad5147 (2016).

82. Chopin MC, Chopin A, Bidnenko E. Phage abortive infection in lactococci: variations on a theme. Curr. Opin. Microbiol. 8(4),473-479 (2005).

83. Goldfarb T, Sberro H, Weinstock E et al. BREX is a novel phage resistance system widespread in microbial genomes EMBO J. 34(2), 169-183 (2015).

84. Chinenova TA, Mkrtumian NM, Lomovskaia ND. Genetic characteristics of a new phage resistance trait in Streptomyces coelicolor A3(2). Genetika 18(12), 1945-1952 (1982).

85. León M, Bastías R. Virulence reduction in bacteriophage resistant bacteria. Front. Microbiol. 6, 343 (2015).

86. Oechslin F, Piccardi P, Mancini S et al. Synergistic interaction between phage therapy and antibiotics clears Pseudomonas aeruginosa infection in endocarditis and reduces virulence. J. Infect. Dis. 215, 703-712 (2017).

87. Orndorff PE. Use of bacteriophage to target bacterial surface structures required for virulence: a systematic search for antibiotic alternatives. Curr. Genet. 62(4), 753-757 (2016).

88. Chan BK, Sistrom M, Wertz JE, Kortright KE, Narayan D, Turner PE. Phage selection restores antibiotic sensitivity in MDR Pseudomonas aeruginosa. Sci. Rep. 6, 26717 (2016).

89. Nale JY, Spencer J, Hargreaves KR et al. Bacteriophage combinations significantly reduce Clostridium difficile growth in vitro and proliferation in vivo. Antimicrob. Agents Chemother. 60(2), 968-981 (2016).

90. Fischer S, Kittler S, Klein G, Glünder G. Impact of a single phage and a phage cocktail application in broilers on reduction of Campylobacter jejuni and development of resistance. PLoS ONE 8(10), e78543 (2013).

91. Fu W, Forster T, Mayer O, Curtin JJ, Lehman SM, Donlan RM. Bacteriophage cocktail for the prevention of biofilm formation by Pseudomonas aeruginosa on catheters in an in vitro model system. Antimicrob. Agents Chemother. 54(1) 397-404 (2010).

92. O’ Flynn G, Ross RP, Fitzgerald GF, Coffey A. Evaluation of a cocktail of three bacteriophages for biocontrol of Escherichia coli O157:H7. Appl. Environ. Microbiol. 70(6), 3417-3424 (2004).

93. Górski A, Międzybrodzki R, Weber-Dąbrowska B et al. Phage therapy: combating infections with potential for evolving from merely a treatment for complications to targeting diseases. Front. Microbiol. 7, 1515 (2016).

94. Mattila S, Ruotsalainen P, Jalasvuori M. On-demand isolation of bacteriophages against drug- resistant bacteria for personalized phage therapy. Front. Microbiol. 6, 1271 (2015). 
95. O'Flaherty S, Ross RP, Meaney W, Fitzgerald GF, Elbreki MF, Coffey A. Potential of the polyvalent anti-staphylococcus bacteriophage K for control of antibiotic-resistant staphylococci from hospitals. Appl. Environ. Microbiol. 71(4), 1836-1842 (2005).

96. Friman VP, Soanes-Brown D, Sierocinski P et al. Pre-adapting parasitic phages to a pathogen leads to increased pathogen clearance and lowered resistance evolution with Pseudomonas aeruginosa cystic fibrosis bacterial isolates. J. Evol. Biol. 29(1), 188-198 (2016).

97. Morello E, Saussereau E, Maura D, Huerre M, Touqui L, Debarbieux L. Pulmonary bacteriophage therapy on Pseudomonas aeruginosa cystic fibrosis strains: first steps towards treatment and prevention. PLoS ONE 6(2), e16963 (2011).

98. Mapes AC, Trautner BW, Liao KS, Ramig RF. Development of expanded host range phage active on biofilms of multi-drug resistant Pseudomonas aeruginosa. Bacteriophage 6(1), e1096995 (2016).

- Describes a method to expand the host range of phage.

99. Ross A, Ward S, Hyman P. More is better: selecting for broad host range bacteriophages. Front. Microbiol. 7, 1352 (2016).

100. Gu J, Liu X, Li Y et al. A method for generation phage cocktail with great therapeutic potential. PLoS ONE 7(3), e31698 (2012).

101. Raya RR, Oot RA, Moore-Maley B et al. Naturally resident and exogenously applied T4-like and T5-like bacteriophages can reduce Escherichia coli O157:H7 levels in sheep guts. Bacteriophage 1, 15-24 (2011).

102. Gill JJ, Hyman P. Phage choice isolation and preparation for phage therapy. Curr. Pharm. Biotechnol. 11(1), 2-14 (2010).

103. Tawil N, Sacher E, Mandeville R, Meunier M. Bacteriophages: biosensing tools for multi-drug resistant pathogens. Analyst 139(6), 1224-1236 (2014).

104. Denyes JM, Dunne M, Steiner S et al. Modified bacteriophage S16 long tail fiber proteins for rapid and specific immobilization and detection of Salmonella cells. Appl. Environ. Microbiol. 83(12), e00277-17 (2017).

105. Bárdy P, Pantůček R, Benšík M, Doškař J. Genetically modified bacteriophages in applied microbiology. J. Appl. Microbiol. 121(3), 618-633 (2016).

106. Expert Round Table on Acceptance and Re-Implementation of Bacteriophage Therapy. Silk route to the acceptance and reimplementation of bacteriophage therapy. Biotechnol. J. 11(5), 595-600 (2016).

107. Kirby AE. Synergistic action of gentamicin and bacteriophage in a continuous culture population of Staphylococcus aureus. PLoS ONE 7(1), e51017 (2012).

108. Davies JK. Genomics and recombination. In: Neisseria, Molecular Mechanisms of Pathogenesis. Genco CA, Wetzler L (Eds). Caister Academic Press, Norfolk, UK, 41-51 (2010).

109. Piekarowicz A, Kłyz A, Majchrzak M, Stein DC. Oral immunization of rabbits with S. enterica Typhimurium expressing Neisseria gonnorrhoeae filamentous phage $\Phi 6$ induces bacterial antibodies against $N$. gonnorrhoeae. Sci. Rep. 6, 22549 (2016).

- Details the potential effectiveness of utilizing a Neisseria gonnorrhoeae prophage sequence for vaccine development against the antibiotic-resistant pathogen.

110. O' Flaherty S, Coffey A, Meaney WJ, Fitzgerald GF, Ross RP. Inhibition of bacteriophage K proliferation on Staphylococcus aureus in raw bovine milk. Lett. Appl. Microbiol. 41(3), 274-279 (2005).

111. Górski A, Dąbrowska K, Międzybrodzki R et al. Phages and immunomodulation. Future Microbiol. 12(10), 905-914 (2017).

112. Górski A, Jonczyk-Matysiak E, Łusiak-Szelachowska M, Międzybrodzki R, Weber-Dąbrowska B, Borysowski J. Bacteriophages targeting intestinal epithelial cells: a potential novel form of immunotherapy. Cell. Mol. Life Sci. 75(4), 589-595 (2017).

113. Reyes A, Semenkovich NP, Whiteson K, Rohwer F, Gordon JI. Going viral: next-generation sequencing applied to phage populations in the human gut. Nat. Rev. Microbiol. 10, 607-617 (2012).

114. Międzybrodzki R, Borysowski J, KIłak M et al. In vivo studies on the influence of bacteriophage preparations on the autoimmune inflammatory process. Biomed. Res. Int. 2017, 3612015 (2017).

115. Akimkin VG, Kalmykov AA, Aminev RM, Polyakov VS, Artebyakin SV. Experience of using bacteriophage and bitilisin-5 to reduce the incidence of respiratory diseases of bacterial ethiology in military personnel. Voen. Med. Zh. 337(2), 36-40 (2016). 